ARTICLE

DOI: $10.1038 / \mathrm{s} 41467-017-02124-9$

\title{
Identification of regulatory targets for the bacterial Nus factor complex
}

\author{
Gabriele Baniulyte ${ }^{1,2}$, Navjot Singh ${ }^{1}$, Courtney Benoit ${ }^{1}$, Richard Johnson ${ }^{1,2}$, Robert Ferguson ${ }^{1}$, \\ Mauricio Paramo (1) ${ }^{1}$, Anne M. Stringer ${ }^{1}$, Ashley Scott ${ }^{1}$, Pascal Lapierre ${ }^{1} \&$ Joseph T. Wade ${ }^{1,2}$
}

Nus factors are broadly conserved across bacterial species, and are often essential for viability. A complex of five Nus factors (NusB, NusE, NusA, NusG and SuhB) is considered to be a dedicated regulator of ribosomal RNA folding, and has been shown to prevent Rhodependent transcription termination. Here, we identify an additional cellular function for the Nus factor complex in Escherichia coli: repression of the Nus factor-encoding gene, suhB. This repression occurs primarily by translation inhibition, followed by Rho-dependent transcription termination. Thus, the Nus factor complex can prevent or promote Rho activity depending on the gene context. Conservation of putative NusB/E binding sites upstream of Nus factor genes suggests that Nus factor autoregulation occurs in many bacterial species. Additionally, many putative NusB/E binding sites are also found upstream of other genes in diverse species, and we demonstrate Nus factor regulation of one such gene in Citrobacter koseri. We conclude that Nus factors have an evolutionarily widespread regulatory function beyond ribosomal RNA, and that they are often autoregulatory.

\footnotetext{
${ }^{1}$ Wadsworth Center New York State Department of Health, Albany, NY 12208, USA. ${ }^{2}$ Department of Biomedical Sciences, School of Public Health University at Albany, Rensselaer, NY 12144, USA. Correspondence and requests for materials should be addressed to J.T.W. (email: joseph.wade@health.ny.gov)
} 
$\mathrm{N}$ us factors are widely conserved in bacteria and play a variety of important roles in transcription and translation $^{1}$. The Nus factor complex comprises the four classical Nus factors, NusA, NusB, NusE (ribosomal protein S10), NusG, and a recently discovered member, SuhB. As a complex, Nus factors serve an important role in promoting expression of ribosomal RNA (rRNA) ${ }^{2,3}$. A NusB/E complex binds BoxA sequence elements in nascent rRNA, upstream of the $16 \mathrm{~S}$ and $23 \mathrm{~S}$ genes $^{4,5}$. Once bound to BoxA, NusB/E has been proposed to interact with elongating RNAP via the NusE-NusG interaction ${ }^{6}$. The role of NusA in Nus complex function is unclear, but may involve binding of NusA to RNA flanking the BoxA ${ }^{7}$. NusA has also been proposed to be a general Rho antagonist by competing with Rho for RNA sites ${ }^{8}$. Early studies of Nus factors focused on their role in preventing both Rho-dependent and intrinsic termination of $\lambda$ bacteriophage RNAs ('antitermination') ${ }^{9}$, which is completely dependent on the bacteriophage protein N. Nus factors can prevent Rho-dependent termination in the absence of $\mathrm{N}^{10,11}$, and for many years, Nus factors were believed to prevent Rho-dependent termination of $\mathrm{rRNA}^{9}$. However, it was recently shown that rRNA is intrinsically resistant to Rho termination, and that the primary role of Nus factors at rRNA is to promote proper RNA folding during ribosome assembly ${ }^{3,12}$.

The most recently discovered Nus factor, SuhB, has been proposed to stabilize interactions between the NusB/E-bound BoxA and elongating RNAP, thus contributing to proper folding of $\mathrm{rRNA}^{12}$. Genome-wide approaches revealed that $\operatorname{suhB}$ is upregulated in the presence of the Rho inhibitor bicyclomycin, suggesting that $\operatorname{suh} B$ is subject to premature Rho-dependent transcription termination ${ }^{13,14}$. Surprisingly, $\operatorname{suh} B$ is also one of the most upregulated genes in $\Delta n u s B$ cells ${ }^{12}$, suggesting a possible autoregulatory function for Nus factors. Moreover, autoregulation of $\operatorname{suh} B$ has been suggested previously ${ }^{15}$, although the mechanism for this regulation is unclear.

Here, we show that $\operatorname{suhB}$ is translationally repressed by Nus factors, which in turn leads to premature Rho-dependent transcription termination. This represents a novel mechanism for control of premature Rho-dependent termination, and is the first described cellular function for Nus factors beyond regulation of rRNA. Moreover, the role of Nus factors at $s u h B$ is to promote Rho-dependent termination of $\operatorname{suhB}$, in contrast to their established function in antagonizing Rho. Bioinformatic analysis suggests that regulation by Nus factors is widespread, and that autoregulation of genes encoding Nus factors, in particular suhB, is a common phenomenon. We confirm Nus factor association with $s u h B$ mRNA in Salmonella enterica, and we demonstrate Nus factor regulation of an unrelated gene in Citrobacter koseri. Thus, our data show that Nus factors are important regulators with diverse targets and diverse regulatory mechanisms.

\section{Results}

Rho-dependent termination within the suhB gene. Genomewide analysis of Rho termination events suggested Rhodependent termination within the Escherichia coli suhB gene $^{13,14}$. To confirm this, we used Chromatin Immunoprecipitation (ChIP) coupled with quantitative PCR (ChIP-qPCR) to determine RNAP association across the $\operatorname{suhB}$ gene in wild-type cells and cells expressing a mutant Rho (R66S) that has impaired termination activity, likely due to a defect in RNA loading ${ }^{16}$. In wild-type cells, we observed a large decrease in RNAP association at the $3^{\prime}$ end of $\operatorname{suhB}$ relative to the $5^{\prime}$ end. This decrease was substantially reduced in rho mutant cells (Fig. 1). Thus, our ChIP data independently support the observation of Rho termination within $\operatorname{suh} B^{13,14}$.

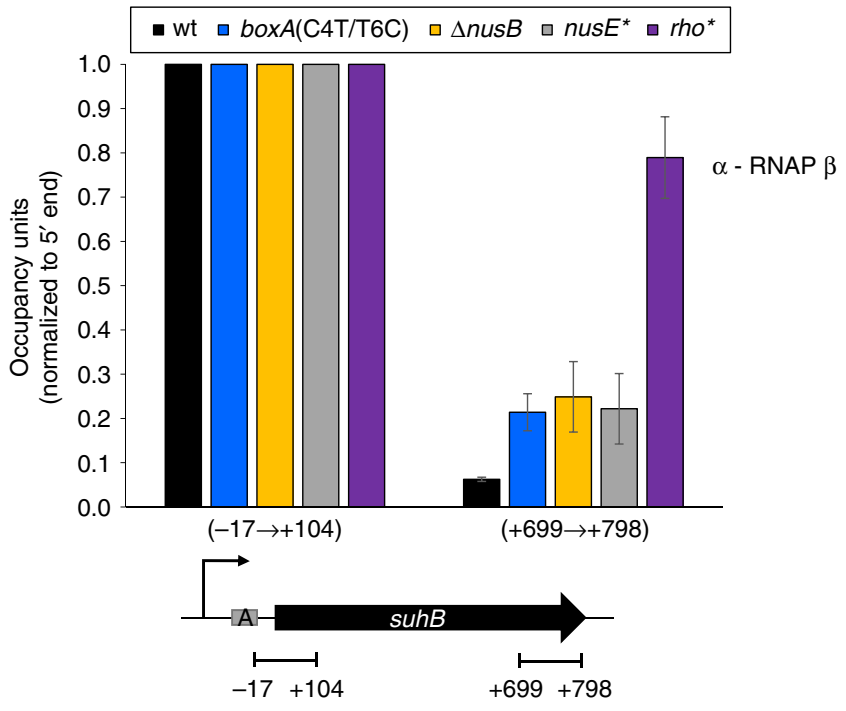

Fig. 1 Transcription termination within suhB is dependent on Rho and Nus factors. RNAP ( $\beta$ ) enrichment at suhB $5^{\prime}$ and $3^{\prime}$ regions was measured using ChIP-qPCR in wild-type MG1655, boxA(C4T/T6C), $\Delta$ nusB, nusE(A12E) or $r$ ho(R66S) mutant strains. Values are normalized to signal at the $5^{\prime}$ end of suhB. $x$-axis labels indicate qPCR amplicon position relative to suhB. Error bars represent \pm 1 standard deviation from the mean $(n=3)$. A schematic depicting the suhB gene, the transcription start site (bent arrow) and box $A$ (grey rectangle) is shown below the graph. Horizontal black lines indicate the position of PCR amplicons

Nus factors are trans-acting regulators of suhB. Based on an approach used to identify modulators of Rho-dependent termination within $S$. enterica chi $P^{17}$, we used a genetic selection to isolate 30 independent mutants defective in Rho-dependent termination within $\operatorname{suhB}$ (see Methods). All 30 strains isolated had a mutation in one of three genes: nusB (14 mutants), nusE (13 mutants) or $n u s G$ (3 mutants) (Supplementary Table 1). We then measured RNAP association across the $s u h B$ gene in wild-type, $\triangle n u s B$ and $n u s E$ mutant cells ( $n u s E$ A12E mutant isolated from the genetic selection). Mutation of $n u s B$ or $n u s E$ increased RNAP binding at the $\operatorname{suh} B 3^{\prime}$ end $\sim 4$-fold compared to wild-type cells (Fig. 1 and Supplementary Fig. 1). We conclude that Nus factors promote Rho-dependent termination within the suhB gene. However, RNAP occupancy at the $3^{\prime}$ end of $\operatorname{suhB}$ in $n u s B$ and $n u s E$ mutants was substantially lower than in the rho mutant (Fig. 1 and Supplementary Fig. 1). This difference may be due to spurious, non-coding transcripts arising from nearby intragenic promoters, which are widespread in $E$. coli ${ }^{18}$ and are often terminated by $\mathrm{Rho}^{13,14}$.

A functional BoxA in the $\operatorname{suhB} 5^{\prime}$ UTR. We identified a sequence in the $\operatorname{suhB} 5^{\prime}$ UTR with striking similarity to boxA sequences from rRNA loci (Supplementary Table 2). Moreover, this boxA-like sequence is broadly conserved across Enterobacteriaceae species (Fig. 2a and Supplementary Fig. 2), suggesting that it is a genuine binding site for $\mathrm{NusB} / \mathrm{E}$. We generated a library of randomly mutated suhB-lac $Z$ transcriptional fusions (see Methods), and identified fusions that had higher expression of lacZ. All identified mutants carried a single nucleotide change at one of five different positions within the putative boxA (Fig. 2b). We then constructed a strain carrying two chromosomal point mutations in the putative $\operatorname{suhB}$ boxA (C4T/T6C; numbers corresponding to the position in the consensus boxA; Supplementary Table 2). We used ChIP-qPCR to measure association of FLAG-tagged SuhB at the $5^{\prime}$ end of the $\operatorname{suhB}$ gene in 


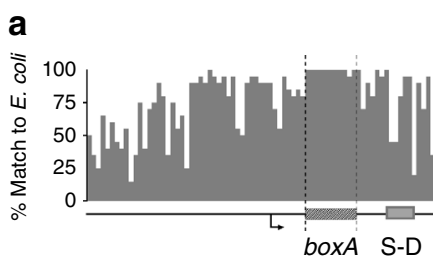

C

b
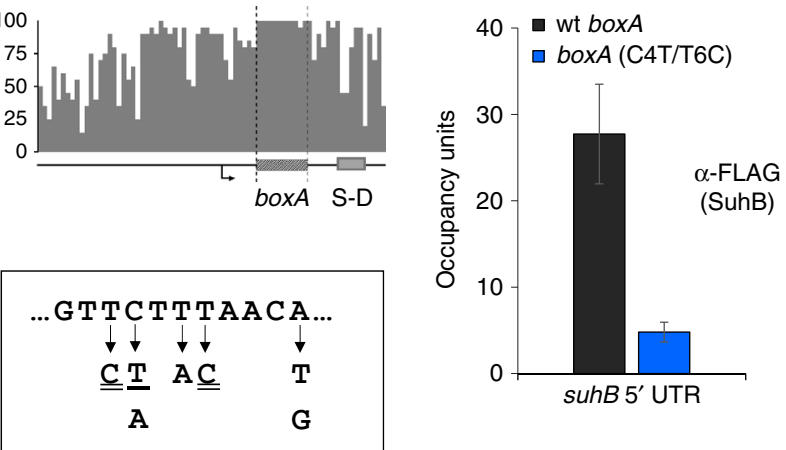

Fig. 2 A functional BoxA in the $5^{\prime}$ UTR of suhB. a Sequence conservation of the $100 \mathrm{bp}$ upstream of suhB and its homologues across 20

Enterobacteriaceae species. The transcription start site is indicated by a bent arrow, and the BoxA and S-D sequences are indicated. $\mathbf{b}$ List of boxA mutations that are associated with increased suhB expression. All single nucleotide changes are indicated by an arrow. Single underline indicates a mutation that was isolated in the absence of mutations anywhere else in the cloned region; other mutants included additional mutations outside the boxA. Double underline indicates that the boxA mutation was isolated in two or more independent clones. Critical position ' -4 ' is indicated (see Supplementary Table 2). c SuhB association with the $5^{\prime}$ end of suhB in wildtype ('wt') and boxA mutant ('boxA C4T/T6C') strains. SuhB-FLAG

occupancy was measured by ChIP-qPCR using $\alpha$-FLAG antibody. Error bars represent \pm 1 standard deviation from the mean $(n=3)$

wild-type cells, or cells containing the boxA mutation. We detected robust association of SuhB-FLAG in wild-type cells, but not in the boxA mutant strain (Fig. 2c). We conclude that the putative BoxA in the $5^{\prime}$ UTR of $\operatorname{suhB}$ is genuine, and recruits Nus factors. To test whether the BoxA controls Rho-dependent termination within $s u h B$, we measured RNAP occupancy across suhB in the boxA mutant strain. We detected a $\sim 4$-fold increase in RNAP occupancy in the downstream portion of $s u h B$ in the boxA mutant strain relative to wild-type cells, mirroring the effect of mutating $n u s B$ or $n u s E$ (Fig. 1). Our data support a model in which Nus factor recruitment by the suhB BoxA leads to Rhodependent termination within the gene.

Nus factors mediate translational repression of suhB. The $\operatorname{suhB}$ BoxA is separated by only $6 \mathrm{nt}$ from the Shine-Dalgarno (S-D) sequence (Fig. 2a). Rho cannot terminate transcription of translated RNA, likely because RNAP-bound NusG interacts with ribosome-associated NusE (S10) ${ }^{6}$. Hence, we hypothesized that NusB/E association with BoxA sterically blocks association of the $30 \mathrm{~S}$ ribosome with the mRNA, repressing translation initiation, uncoupling transcription and translation, and thereby promoting Rho-dependent termination. To test this hypothesis, we used the suhB-lacZ transcriptional fusion (Fig. 3a), as well as an equivalent translational fusion (Fig. 3b). We reasoned that mutation of $n u s B$, $n u s E$ or boxA would result in increased expression from both reporter fusions, since these mutations would relieve translational repression (reported by the translational fusion), which in turn would reduce Rho-dependent termination (reported by the transcriptional fusion). In contrast, we reasoned that mutation of rho would result in increased expression only from the transcriptional fusion reporter, since the SuhB-LacZ fusion protein (from the translational fusion construct) would still be translationally repressed. We measured expression of lac $Z$ from each of these reporter fusions in wild-type cells, and cells with $\Delta n u s B$, nusE A12E or rho R66S mutations. We also measured expression of lacZ in these strains using reporter fusions carrying the C4T/
T6C boxA mutation. Consistent with our model, we detected increased expression of both reporter fusion types in mutants of $n u s B$, nusE or boxA, whereas mutation of rho resulted in increased expression of the transcriptional fusion but not the translational fusion reporter (Fig. 3a, b). Note that mutation of $n u s B, n u s E$ or boxA does not lead to the same level of increase in expression of the reporter fusions (Fig. 3a, b). This is likely due to the fact that mutations in Nus factors have extensive pleiotropic effects, presumably due to the importance of Nus factors in ribosome assembly ${ }^{3}$. Moreover, mutation of boxA in a nusE mutant leads to a further increase in reporter expression, whereas mutation of boxA in a nusB mutant does not (Fig. 3a, b). This is likely due to the mutant NusE retaining partial function, whereas deletion of $n u s B$ completely abolishes Nus factor function.

To confirm the effects of mutating nusB, nusE, rho and boxA on expression of $\operatorname{suhB}$ in the native context, we measured SuhB protein levels by western blotting using strains expressing a Cterminally FLAG-tagged derivative of SuhB. We compared SuhB protein levels in cells with nusE A12E, rho R66S or boxA C4T/ T6C mutations; we have previously shown that SuhB protein levels are increased in a $\Delta n u s B$ mutant ${ }^{12}$. SuhB protein levels in the mutant strains correlated well with the translational $\operatorname{suhB} B$ lac $Z$ fusion reporter gene assay: mutation of $n u s E$ or boxA caused a modest increase in SuhB-FLAG levels, whereas mutation of rho had no discernible effect (Fig. 3c, d; Supplementary Fig. 7).

Rho-dependent transcription termination occurs early in the suhB gene. Rho-dependent termination requires a Rho loading sequence known as a Rut that typically occurs $>60$ nt upstream of the termination site(s), is pyrimidine-rich and G-poor ${ }^{19}$. To localize the Rut and the downstream termination site(s), we constructed a short transcriptional suhB-lacZ fusion that includes only the first $57 \mathrm{bp}$ of the $\operatorname{suhB}$ gene. Expression of this reporter fusion was substantially higher in rho mutant cells than in wildtype cells (Fig. 4a). In contrast, expression was only marginally higher in rho mutant cells than in wild-type cells when the boxA sequence was mutated (Fig. 4a). Thus, the short suhB-lacZ reporter fusion behaves similarly to the fusion that includes the entire $s u h B$ gene (Fig. 3a), indicating that the rut and termination sequences must be upstream of position 57 within $s u h B$.

Given that the short suhB-lacZ fusion includes only $94 \mathrm{bp}$ of transcribed sequence from $\operatorname{suhB}$ and its $5^{\prime} \mathrm{UTR}$, and that Rut sequences are typically found $>60 \mathrm{nt}$ from the site(s) of termination $^{19}$, we reasoned that the Rut is likely located close to the $5^{\prime}$ end of the $\operatorname{suhB} 5^{\prime}$ UTR. Consistent with this, positions 2-22 of the $5^{\prime}$ UTR include 17 pyrimidines and only one G. This sequence completely encompasses the box $A$, suggesting that the box $A$ and rut sequences overlap. To determine whether mutation of the boxA affects Rho-dependent termination independent of Nus factor-mediated translational repression, we constructed short suhB-lacZ fusions in which the $\operatorname{suhB}$ start codon was mutated, either alone or in conjunction with a mutated boxA. We reasoned that mutation of the suhB start codon would bypass the need for BoxA-mediated translational repression to cause Rhodependent termination. As expected, expression of the fusion with the mutated start codon but wild-type boxA was substantially higher in a rho mutant than in wild-type cells (Fig. 4a), consistent with this construct being Rho-terminated. However, expression of the fusion with the mutated start codon and mutated boxA was only marginally higher in a rho mutant than in wild-type cells, indicating that Rho-dependent termination is disrupted by mutation of the boxA, even in the absence of suhB translation. We conclude that mutation of the boxA reduces Rhodependent termination by disrupting the rut. This likely occurs due to the boxA and rut sequences overlapping, in which case 
a

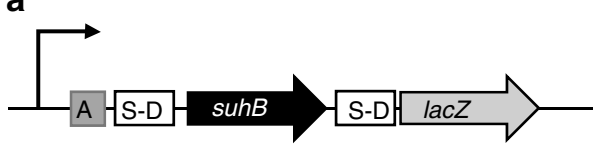

suhB transcriptional fusion

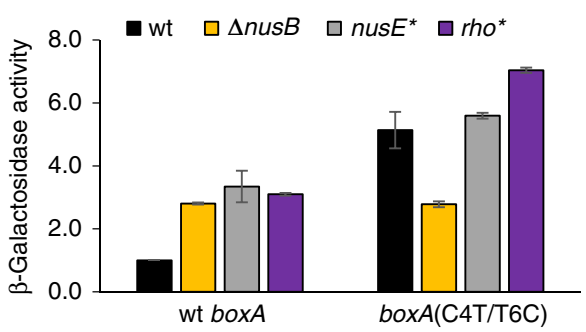

C

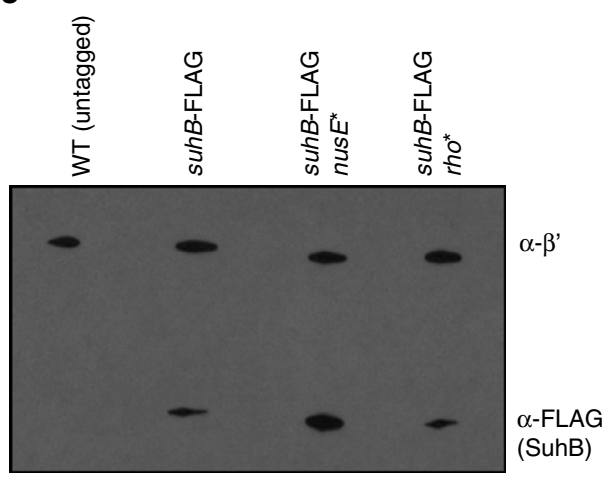

b

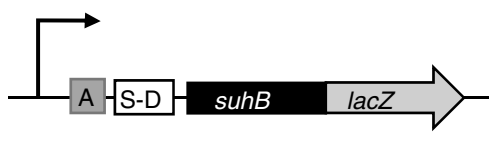

suhB translational fusion

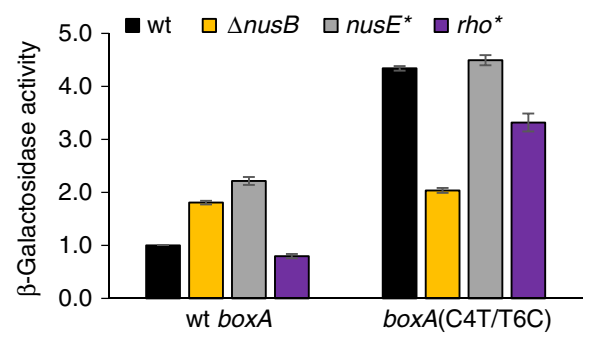

d

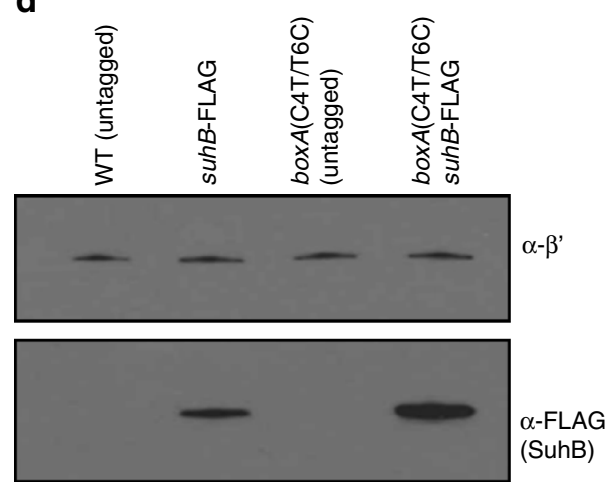

Fig. 3 Nus factors repress translation of suhB, leading to Rho-dependent termination within the gene. $\beta$-galactosidase activity of (a) transcriptional and (b) translational fusions of suhB to lacZ in wild-type cells, $\Delta$ nusB, nusE(A12E), or rho(R66S) mutants. The suhB-lacZ fusion had either a wild-type ('wt') or mutant boxA ('C4T/T6C'). Data are normalized to levels in wild-type cells. Error bars represent \pm 1 standard deviation from the mean ( $n=3$ ). Schematics of constructs used in these experiments are depicted above the graphs. (c) and (d) Western blots showing SuhB-FLAG protein levels in wild-type cells, nusE (A12E), rho(R66S) c, and boxA(C4T/T6C) mutants d. SuhB-FLAG was probed with $\alpha$-FLAG antibody; RNAP $\beta^{\prime}$ was probed as a loading control.

Representative blots from at least three independent experiments are shown. Images were cropped for clarity; unprocessed western blot images are in Supplementary Fig. 7

mutating the boxA would also alter the rut. However, mutation of the boxA might also alter RNA secondary structure of the Rut.

Mutation of the boxA results in greatly decreased Rhodependent termination of a fusion of the entire $\operatorname{suh} B$ gene to lacZ (Fig. 3a). Although this effect could be due to disruption of a rut overlapping the boxA, we reasoned that there are likely to be additional rut sequences within the suhB ORF. To test this hypothesis, we constructed transcriptional fusions of the entire $\operatorname{suh} B$ gene and $5^{\prime}$ UTR to $l a c Z$ with a mutation in the $\operatorname{suhB}$ start codon, either alone or in conjunction with a mutation in the boxA. For both constructs, expression was substantially higher in a rho mutant than in wild-type cells (Fig. $4 \mathrm{~b}$ ), indicating robust Rho-dependent termination within the $\operatorname{suh} B$ gene, even with a mutated boxA. We conclude that the $\operatorname{suh} B$ gene includes at least one additional rut, and that the effect of mutating the boxA on Rho-dependent termination with a long transcriptional fusion (Fig. 3) is due to loss of Nus factor binding rather than a direct effect on Rho loading.

BoxA-mediated occlusion of the S-D sequence is not due to steric occlusion. The data described above are consistent with a steric occlusion model in which $\mathrm{NusB} / \mathrm{E}$ binding to the BoxA directly prevent $30 \mathrm{~S}$ ribosome association with the S-D sequence. However, other mechanisms of translational repression are also possible. The steric occlusion model predicts that increasing the distance between the boxA and S-D elements would relieve translational repression, and consequently Rho-dependent termination. We constructed suhB-lacZ transcriptional fusions that carried insertions of sizes from 2 to $100 \mathrm{bp}$ between the box $A$ and S-D sequences (see Methods for details). We constructed equivalent fusions carrying a boxA mutation (C4A; Supplementary Table 2). Surprisingly, separating the BoxA and S-D sequences with up to $100 \mathrm{nt}$ intervening RNA did not abolish BoxA-mediated repression (Fig. 5). Note that differences in absolute expression levels for the different constructs are likely due to variability in secondary structure around the ribosome binding site. Additionally, we are confident that none of the insertions inadvertently introduces a new promoter, since a similar construct lacking an active upstream promoter was only weakly expressed (Supplementary Fig. 3). We conclude that the steric occlusion model is insufficient to explain BoxA-mediated translational repression of $\operatorname{suh} B$, although the proximity of the BoxA and S-D sequences suggests that simple occlusion would prevent ribosome binding.

We reasoned that if steric occlusion of ribosomes by NusB/E binding is sufficient for repression of $\operatorname{suhB}$, it would not require assembly of a complete Nus factor complex, since NusB/E alone has a high affinity for BoxA RNA ${ }^{4}$. Hence, we constructed suhBlac $Z$ translational fusions where the native promoter is replaced by a T7 promoter. Previous studies showed that gene regulation 
a
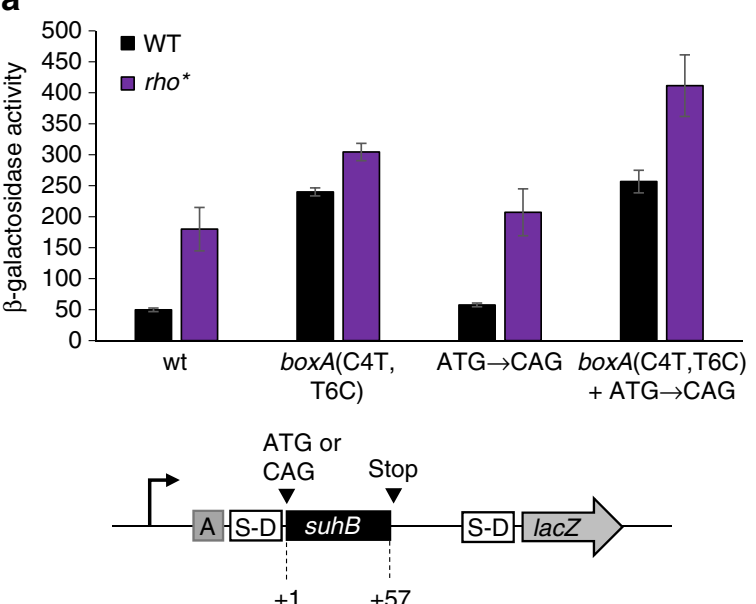

b

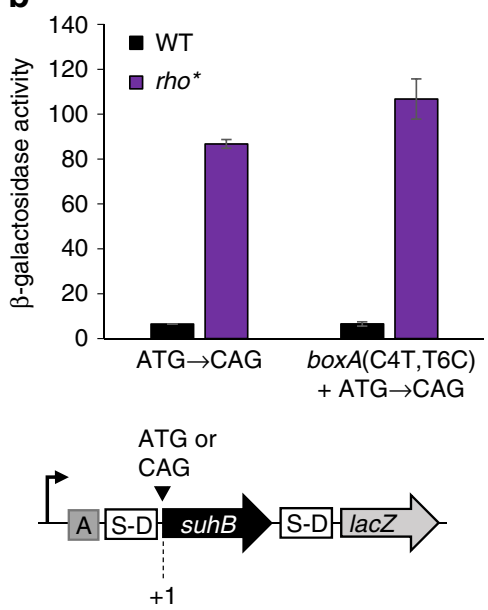

Fig. 4 The suhB BoxA overlaps the first of multiple Rut elements. $\beta$-galactosidase activity of short (a) and full-length (b) suhB transcriptional fusions to lacZ. Constructs included either a wild-type sequence ('wt'), boxA mutation ('C4T, T6C') and/or suhB start codon mutation ('ATG $\rightarrow$ CAG'), as indicated on the $x$-axis. $200 \mathrm{bp}$ of sequence upstream of the suhB gene was included in all constructs. Error bars represent \pm 1 standard deviation from the mean ( $n=3$ ). Schematics of the constructs used for these experiments are depicted below the graphs

involving $\lambda \mathrm{N}$ or NusG is lost when $E$. coli RNAP is substituted with bacteriophage T7 RNAP ${ }^{20-22}$, suggesting that T7 RNAP does not interact with Nus factors; hence, transcription of this suhB-lacZ fusion by T7 RNAP would not be associated with the formation of a complete Nus factor complex. We grew cells at $37^{\circ}$ C, $30^{\circ} \mathrm{C}$ or room temperature $\left(23^{\circ} \mathrm{C}\right)$, since the transcription elongation rate of T7 RNAP is similar to that of E. coli RNAP at room temperature, but considerably higher at $37^{\circ} \mathrm{C}^{23,24}$. At all temperatures, we detected robust expression that was dependent upon expression of T7 RNAP in the same cells. However, we observed no effect on expression of mutating the boxA (Supplementary Fig. 4). We conclude that efficient BoxAdependent repression of $\operatorname{suh} B$ requires assembly of a complete Nus factor complex.

Salmonella enterica suhB has a functional BoxA. Phylogenetic analysis of the region upstream of the $s u h B$ gene indicates that the boxA sequence is widely conserved among members of the family Enterobacteriaciae (Fig. 2a; Supplementary Table 2; Supplementary Fig. 2), suggesting that BoxA-mediated regulation of suhB occurs in these species. To investigate this possibility, we used ChIP of FLAG-tagged SuhB to measure association of SuhB with the $s u h B$ upstream region in $S$. enterica subspecies enterica serovar Typhimurium. We detected robust association of both RNAP ( $\beta$ subunit) and SuhB with the $\operatorname{suhB}$ upstream region (Supplementary Fig. 5), indicating that the $s u h B$ mRNA contains a functional BoxA. We also failed to detect association with a previously reported cryptic BoxA within the his $G$ gene (Supplementary Fig. 5), consistent with the sequence of this element differing at a critical position from the BoxA consensus (Supplementary Table 2).

BoxA-mediated regulation and Nus factor autoregulation are phylogenetically widespread. Aside from their role in lambdoid phage, Nus factors have historically been considered dedicated regulators of rRNA expression. Our discovery of $\operatorname{suhB}$ as a novel regulatory target of Nus factors suggests that BoxA-mediated regulation may be more extensive. BoxA sequences in rRNA are known to be highly conserved ${ }^{2}$. Based on the boxA sequences from $E$. coli rRNA and $\operatorname{suh} B$ loci, and a previous analysis of sequences required for BoxA function in $E$. coli ${ }^{5}$, we derived a consensus sequence (GYTCTTTAANA) that is likely to be
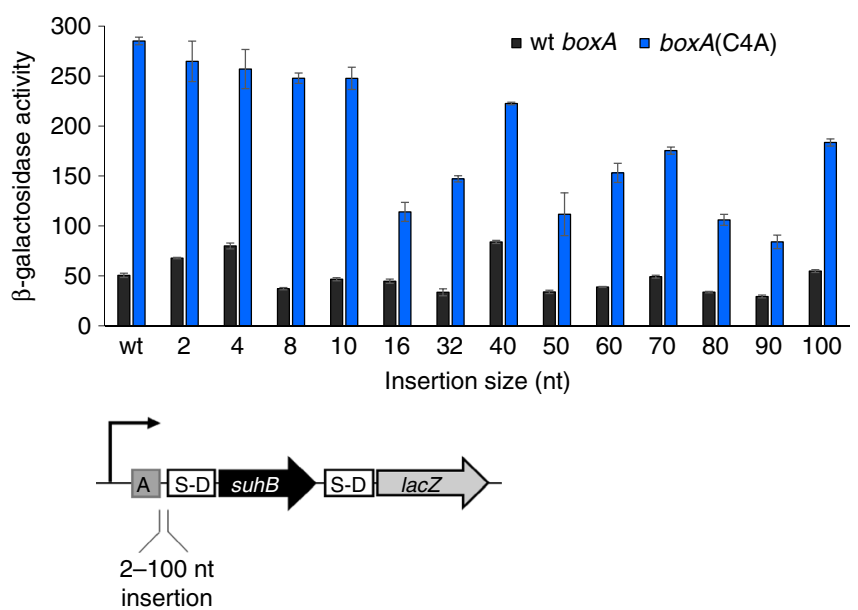

Fig. 5 The effect on suhB-lacZ transcription levels of altering the distance between box $A$ and the S-D sequence. $\beta$-galactosidase activity of wild-type ('wt boxA'; dark grey bars) and boxA mutant ('C4A'; blue bars)

transcriptional fusions of suhB to $l a c Z$, with increasing lengths of non-coding DNA inserted between the box $A$ and $S-D$ sequences. The length of inserted sequence (nt) is indicated on the $x$-axis. Constructs include 200 bp of upstream sequence and a full-length suhB fused to lac $Z$ in the pAMD-BAlac $Z$ plasmid. Note that the sequence of inserted non-coding DNA differs for constructs with insertion sizes of $\leq 32 \mathrm{bp}$ and $\geq 40 \mathrm{bp}$ (see Methods and Supplementary Fig. 6 for details)

applicable to almost all $\gamma$-proteobacteria ${ }^{2}$. We searched for perfect matches to this sequence in 940 sequenced $\gamma$-proteobacterial genomes. We then selected sequence matches that are positioned within $50 \mathrm{bp}$ of a downstream start codon for an annotated gene. Thus, we identified 407 putative BoxA sequences from 314 genomes, with between 0 and 7 instances per genome (Supplementary Data 1). We determined whether any gene functions were identified from multiple genomes. To minimize biases from the uneven distribution of genome sequences across different genera, we analysed gene functions at the genus rather than species level. Across all the species analysed, we identified 36 different gene functions with at least one representative from one genus. Strikingly, we identified 34 of 55 genera in which at least one species has a putative boxA sequence within $50 \mathrm{bp}$ of the start of 
an annotated $\operatorname{suh} B$ homologue. We identified three additional genera in which at least one species has a putative box $A$ within 50 bp of the start of an unannotated $\operatorname{suh} B$ homologue, and one genus with a species in which the $\operatorname{suh} B$ homologue has a putative boxA $82 \mathrm{bp}$ from the gene start. Thus, our analysis reinforces the notion that BoxA-mediated regulation of $\operatorname{suhB}$ is highly conserved (Fig. 2a and Supplementary Fig. 2). Three other gene functions were represented in multiple genera: prsA (encodes ribosephosphate pyrophosphokinase) and rpsJ (encodes NusE) were each found in three genera, and genes encoding ParE-like toxins were found in two genera. We also identified two genera with species in which rpsJ is predicted to be a downstream gene in an operon where the first gene in the operon has a putative box $A<$ $50 \mathrm{bp}$ from the gene start.

BoxA-mediated regulation of a toxin-antitoxin system in Citrobacter koseri. Bioinformatic analysis strongly suggested that BoxA-mediated regulation is evolutionarily widespread and extends to genes other than $\operatorname{suhB}$. To determine whether Nus factors regulate genes other than rRNA and $s u h B$ in other species, we selected one putative BoxA-regulated gene identified by the bioinformatic search for boxA-like sequences: CKO_00699 from C. koseri (Supplementary Table 2). CKO_00699 is predicted to encode a ParE-like toxin, part of a putative toxin-antitoxin pair. A putative boxA was observed upstream of a homologous gene in Pasteurella multocida, suggesting conserved BoxA-mediated regulation. We reasoned that if CKO_00699 is a genuine target of Nus factors, it would likely retain this regulation in $E$. coli, since Nus factors are highly conserved between C. koseri and E. coli (e.g. the amino acid sequence of NusB is $97 \%$ identical and $100 \%$ similar between the two species). Hence, we constructed a transcriptional fusion of $C K O \_00699$ to lacZ and measured expression in E. coli. Note that we included a mutation in CKO_00699 (R82A) to inactivate the predicted toxin activity to prevent growth inhibition. The lac $Z$ fusion included a strong, constitutive promoter $^{25}$, and the sequence from $C$. koseri began at the predicted transcription start site, based on manual analysis of likely promoter sequences (Fig. 6). We measured expression of fusions with wild-type and mutant boxA (C4A) sequences (Supplementary Table 2), in wild-type and $\triangle n u s B$ strains. Mutation of the putative boxA, or deletion of $n u s B$ resulted in a substantial increase in expression, whereas mutation of the box $A$ did not affect expression in the $\Delta n u s B$ strain (Fig. 6). We conclude that CKO_00699 is directly repressed by a BoxA and Nus factors.

\section{Discussion}

We have shown that premature Rho-dependent termination within the $\operatorname{suhB}$ gene is controlled by a BoxA and Nus factors. This likely serves as a mechanism for autoregulation of Nus factors, since SuhB is a critical component of the Nus machinery ${ }^{12}$. Premature Rho-dependent termination of mRNAs has been recently recognized to be a widespread regulatory mechanism $^{26,27}$. Most regulation of this type occurs by alteration of mRNA accessibility around Rut sites. In the case of $\operatorname{suhB}$, Rhodependent termination occurs as a result of translational repression.

A function for Nus factors in promoting Rho-dependent termination is particularly striking because of their long association with antitermination ${ }^{9}$. The contrasting effects of Nus factors on Rho-dependent termination in different contexts, and their role in promoting ribosomal assembly, highlight the flexibility in the function of these proteins. Our data indicate that translational repression of $s u h B$ by Nus factors is not due to occlusion of the SD. Previous studies of Nus factors suggest that they form a loop between the BoxA in the RNA and the elongating RNAP ${ }^{3,12}$. We
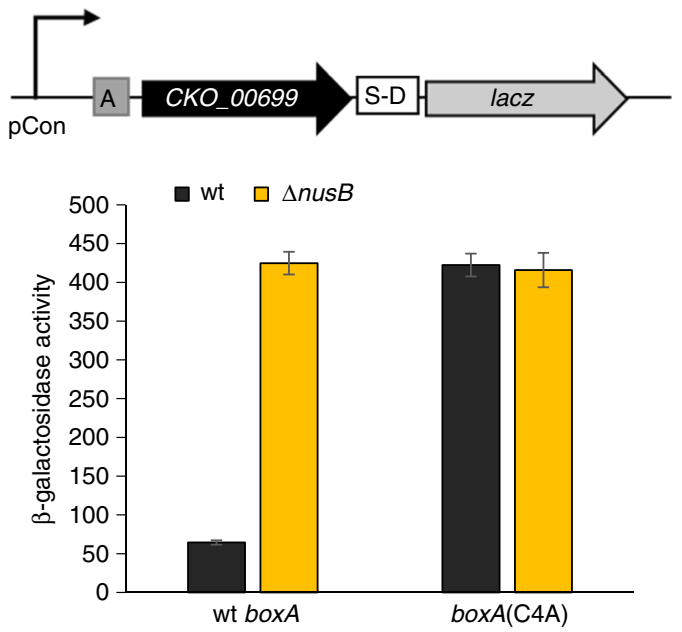

Fig. 6 Identification of a BoxA element in Citrobacter koseri. $\beta$-galactosidase activity of wild-type ('wt boxA') and boxA mutant ('C4A') transcriptional fusions of CKO_00699 (R82A mutant, to avoid potential toxicity to E. coli in the absence of the anti-toxin) to lacZ in E. coli wild-type ('wt'; dark grey bars) or nusB deletion ( $\triangle$ nusB"; yellow bars) strains. $C K O \_00699-l a c Z$ transcription was driven by a constitutive promoter ${ }^{25}$. Error bars represent \pm 1 standard deviation from the mean $(n=3)$

propose that this loop prevents the $30 \mathrm{~S}$ ribosome from accessing the S-D. Alternatively, association of NusG with NusE in the context of the Nus factor complex may prevent translation by blocking association of NusG with ribosome-associated NusE (S10).

Autoregulation of SuhB is strikingly similar to autoregulation of $\lambda \mathrm{N}$. $\lambda$ nutL is positioned $\sim 200 \mathrm{bp}$ upstream of the $N$ gene. Binding of Nus factors and $\mathrm{N}$ to NutL results in translational repression of $\mathrm{N}^{28}$. The distance between NutL and the S-D sequence is such that a simple steric occlusion model is insufficient to explain translational repression by $\mathrm{N}$ and Nus factors; the RNA loop formed between NutL and the elongating RNAP provides a straightforward explanation of repression. Although the gap between NutL and the S-D sequence for the $N$ gene is considerably longer than the longest distance we tested for $\operatorname{suhB}$ (Fig. 5), the intervening sequence is highly structured ${ }^{29}$, which may impact the compactness of the loop.

Although we have shown previously that Nus factors are not required to prevent Rho-dependent termination at $\mathrm{rRNA}$ loci ${ }^{12}$, Nus factors have been shown to prevent Rho-dependent termination in artificial reporter constructs ${ }^{10,11,30,31}$. Our finding that Nus factors promote Rho-dependent termination in $s u h B$ further indicates that context determines the precise function of Nus factors. Hence, it is likely that there are additional sequence elements in $\operatorname{suhB}$ that promote Rho-dependent termination, or that there are additional sequence elements in the artificial reporter constructs that prevent Rho-dependent termination.

Our data support a widespread regulatory role for Nus factors, implicating them in regulation in both a wide range of species, and of a diverse set of genes, although within any given species there are likely only a few regulatory targets. Strikingly, $\sim 25 \%$ of the gene functions associated with an upstream boxA are known to be directly connected to translation. This is consistent with the established connection between Nus factors and ribosomal assembly $^{3}$, and suggests that the impact of Nus factors on translation occurs by regulation of a variety of genes. Moreover, our data suggest that NusE is autoregulated in phylogenetically diverse species. Although we did not identify any genomes where genes encoding other Nus factors have putative upstream boxA sequences, we did identify a putative boxA sequence upstream of 
ribH in six different species of Pseudomonas. In all cases, $n u s B$ is the gene immediately downstream of $r i b H$, suggesting that $n u s B$ is autoregulated in pseudomonads. Overall, we identified no species with a putative boxA upstream of more than one Nus factor-encoding gene, and only 11 genera had no putative boxA associated with any Nus factor-encoding gene. However, for five of these latter genera we were unable to identify a boxA sequence upstream of the rRNA genes, suggesting that the BoxA consensus is different to that in E. coli. Thus, our data strongly suggest that Nus factor autoregulation occurs in $\sim 90 \%$ of gammaproteobacterial species, and that typically, just one Nus factor is autoregulated. The evidence for autoregulation of SuhB, NusE and NusB suggests that the levels of these proteins contribute to feedback loops that control the primary function of Nus factors: promoting ribosomal assembly. Our observation of BoxAmediated regulation of a ParE-like toxin in C. koseri demonstrates that Nus factors regulate genes other than their own. Indeed, our bioinformatic analysis suggests that genes of many functions may be regulated by Nus factors, with 36 gene functions represented in at least one genus. Our list is conservative because (i) it does not consider the possibility of regulation by BoxA sequences located $>50 \mathrm{nt}$ upstream of the gene start, which we know is possible (Fig. 5), (ii) it does not consider non-coding RNAs, (iii) the BoxA consensus may be different in some of the species analysed, and (iv) gene starts predicted by bioinformatic annotation pipelines may be incorrect ${ }^{32}$.

Our data indicate that regulation by Nus factors extends to many genes beyond rRNA, and that Nus factor autoregulation is an evolutionarily widespread phenomenon. Moreover, we have shown that Nus factors can provide contrasting forms of regulation, depending on the context of the target; despite their longestablished function in antitermination ${ }^{9}$, Nus factors promote Rho-dependent termination within suhB. Key questions about the function of Nus factors remain to be addressed. What is the molecular architecture of the Nus factor machinery? What are the specific RNA sequences that determine whether Nus factors prevent Rho-dependent termination? How do Nus factors modulate the function of elongating RNAP? Our identification of novel Nus factor target genes with novel regulatory mechanisms provides an excellent opportunity to address these questions.

\section{Methods}

Strains and plasmids. All strains, plasmids and oligonucleotides used in this study are listed in Supplementary Tables 3 and 4. Mutations in rpsJ and rho were P1 transduced into MG1655 ${ }^{33}$ MG1655 SlacZ (AMD054) ${ }^{34}$ and MG1655suhB-FLAG 3 $(\mathrm{VS066})^{12}$. E. coli MG1655suhB(boxA(C4T/T6C)), MG1655suhB(boxA(C4T/ T6C)) $-\mathrm{FLAG}_{3}$ and $S$. Typhimurium his $G \Delta+3:$ thy $A$, his $G \Delta+100::$ thy A suhB-FLAG strains were constructed using the 'Flexible Recombineering Using Integration of thy A' (FRUIT) method ${ }^{35}$

Plasmids pGB1-pGB36, pGB67-68 were constructed by cloning the suhB gene and $200 \mathrm{bp}$ of upstream sequence into the pAMD-BA-lacZ plasmid $^{34}$, creating transcriptional or translational fusions to lacZ. Plasmids pGB192-pGB193 included $200 \mathrm{bp}$ of upstream sequence and $57 \mathrm{nt}$ of $\operatorname{suhB}$ coding sequence followed by a stop codon. Fusions carrying boxA mutations were made by amplifying a $\operatorname{suh} B$ fragment from GB023 (boxA(C4T/T6C)) or by site-directed mutagenesis (boxA(C4A)); suhB start codon mutations (ATG $\rightarrow \mathrm{CAG}$ ) were made using site-directed mutagenesis. Insertions between the boxA and S-D sequences were generated by cloning fragments of random non-coding sequence

('GAACTACCCATCTGGTCGCAGATAGTATGAAC'), modified from ref. ${ }^{36}$, for insertions of up to $32 \mathrm{bp}$; 40-100 bp insertions carried a non-coding sequence from the 16S RNA gene in the reverse orientation (region from +1281 to +1380 ). The $5^{\prime}$ end of the insert remained the same, and inserted sequence was extended towards the S-D sequence (see Supplementary Fig. 6 for details). Plasmid pGB116 was made by cloning the T7 RNAP gene with a S-D sequence into pBAD $18^{37}$. Plasmids pGB83-95 carried the $\operatorname{suhB}$ gene and $36 \mathrm{nt}$ of the 5'UTR with wt or mutant boxA, and a $100 \mathrm{nt}$ insertion between the BoxA and S-D elements, where indicated. suhB was under the control of pT7 promoter and was translationally fused to lac $Z$ reporter on pAMD-BA-lacZ plasmid ${ }^{34}$. Plasmids pGB109-110 were made by cloning CKO_00699(R82A) gene with wt or mutant boxA (C4A) and a constitutive promoter ${ }^{25}$; the toxin gene was transcriptionally fused to lac $Z$ reporter on pAMDBA-lacZ plasmid.

Isolation and identification of trans- and cis-acting mutants. The trans-acting mutant genetic selection was performed using pJTW067 plasmid carrying a suhBlacZ transcriptional fusion in MG1655 $\Delta$ lacZ. pJTW067 was isolated as an inactive suhB-lac $Z$ clone from a cis-acting mutant screen (see below) and contains a single nucleotide mutation (T607C) that does not affect the expression of $\operatorname{suhB}$. It is otherwise identical to pJTW067. Bacterial cultures were grown at $37^{\circ} \mathrm{C}$ in LB medium. One hundred microlitres of an overnight culture was washed and plated on $\mathrm{M} 9+0.2 \%$ lactose agar. Spontaneous survivors were first tested for increased plasmid copy number using $\mathrm{qPCR}$, comparing the $\mathrm{Ct}$ values of plasmid and chromosomal amplicons. Strains with increased copy number were discarded. To eliminate plasmid mutants, plasmids were isolated and transformed into a clean MG1655 $\Delta$ lacZ background and plated on MacConkey agar indicator plates; mutants forming red colonies (upregulated suhB-lacZ) were discarded. Chromosomal mutations were identified either by PCR amplification and sequencing of $n u s B, n u s E$ and $n u s G$, or by whole genome sequencing. Specifically, genomic DNA was purified using a DNeasy blood and tissue kit (Qiagen), DNA libraries were prepared using a Nextera kit (Illumina), sequencing was performed on an Illumina MiSeq instrument, and sequence variants were identified using the CLC genomic workbench (default parameters). The cis-acting mutant genetic screen was performed by cloning a mutant $s u h B$ DNA library, generated by an error-prone DNA polymerase Taq (NEB) with oligonucleotides JW3605 and JW3607, into the pAMD-BA-lacZ vector, which was transformed into EPI300 background (lac'; Epicentre). The mutant library included the entire promoter, $5^{\prime}$ UTR and gene. We selected mutants that were visibly upregulated on MacConkey agar plates and sequenced the insert to identify mutations.

ChIP-qPCR. Bacteria were grown at $37^{\circ} \mathrm{C}$ in $\mathrm{LB}$ medium until $\mathrm{OD}_{600}=0.5-0.6$. ChIP-qPCR was performed as described previously ${ }^{34}$, using monoclonal mouse anti-RpoB (Neoclone \#W0002) and M2 monoclonal anti-FLAG (Sigma) antibodies. Occupancy units represent background-subtracted enrichment scores relative to transcriptionally silent regions within the $b g l B$ or $y n b B$ genes in $E$. coli, and the $s b c C$ gene in $S$. Typhimurium.

$\boldsymbol{\beta}$-galactosidase assays. Bacterial cultures were grown at $37^{\circ} \mathrm{C}$ in $\mathrm{LB}$ medium to an $\mathrm{OD}_{600}$ of $0.5-0.6$. LB medium was supplemented with $0.2 \%$ arabinose when pBAD18 or its derivatives were used. A volume of $100-200 \mu \mathrm{L}$ of culture was pelleted and resuspended in $800 \mu \mathrm{L}$ of $\mathrm{Z}$ buffer $\left(0.06 \mathrm{M} \mathrm{Na}_{2} \mathrm{HPO}_{4}, 0.04 \mathrm{M}\right.$ $\mathrm{NaH}_{2} \mathrm{PO}_{4}, 0.01 \mathrm{M} \mathrm{KCl}, 0.001 \mathrm{M} \mathrm{MgSO}_{4}$ ) supplemented with $\beta$-mercaptoethanol (50 $\mathrm{mm}$ final concentration), sodium dodecyl sulfate $(0.001 \%$ final concentration), and $20 \mu \mathrm{L}$ chloroform. Assays were initiated by adding $160 \mu \mathrm{L}$ of 2 -nitrophenyl $\beta$ D-galactopyranoside $(4 \mathrm{mg} / \mathrm{mL})$ and stopped by adding $400 \mu \mathrm{L}$ of $1 \mathrm{M} \mathrm{Na}_{2} \mathrm{CO}_{3}$. The duration of the reaction and $\mathrm{OD}_{420}$ readings were recorded and $\beta$-galactosidase activity units were calculated as $1000 \times\left(\mathrm{A}_{420} /\left(\mathrm{A}_{600}\right)\left(\right.\right.$ time $\left.\left._{\min }\right)\right)$.

Western blotting. Bacteria were grown at $37^{\circ} \mathrm{C}$ in $\mathrm{LB}$ to an $\mathrm{OD}_{600}$ of $0.5-0.6$. Cel pellets were boiled in gel loading dye, separated on gradient polyacrylamide gels (Bio-Rad), and transferred to a polyvinylidene fluoride membrane (Thermo Scientific). The membrane was cut in half at the $50 \mathrm{kDa}$ molecular marker. The upper part of the membrane was probed with control mouse monoclonal anti-RpoC (BioLegend) antibody at 1:4000 dilution, and the lower part was probed with mouse monoclonal M2 anti-FLAG (Sigma) antibody at 1:10,000 dilution. Goat anti-mouse horseradish peroxidase-conjugated antibody was used for secondary probing at 1:20,000 dilution. Both parts of the membrane were always processed in parallel. Blots were developed with Clarity ${ }^{\mathrm{TM}}$ Western ECL Substrate (Bio-Rad).

Sequence alignment of suhB upstream regions. We extracted $100 \mathrm{bp}$ of upstream sequence for $s u h B$ homologues in 19 species of the family Enterobacteriaciae, and aligned the sequences using MUSCLE ${ }^{38}$ (Supplementary Fig. 2). To determine the \% match to $E$. coli at each position, we added 1 to the number of perfect matches (to account for the E. coli sequence), divided by 20 (to account for the 20 species in the alignment), and converted to a percentage.

Identification of putative boxA sequences in $\boldsymbol{\gamma}$-proteobacterial genomes. We searched all sequenced $\gamma$-proteobacterial genomes for annotated protein-coding genes with the sequence GYTCTTTAANA within the $50 \mathrm{nt}$ upstream of the annotated gene start. We compared gene functions using COG annotations ${ }^{39}$.

Data availability. All relevant data supporting the findings of the study are available in this article and its Supplementary Information files. All computer codes are available from the corresponding author upon request. 
Received: 16 December 2016 Accepted: 8 November 2017

Published online: 11 December 2017

\section{References}

1. Peters, J. M., Vangeloff, A. D. \& Landick, R. Bacterial transcription terminators: the RNA 3'-end chronicles. J. Mol. Biol. 412, 793-813 (2011).

2. Arnvig, K. B. et al. Evolutionary comparison of ribosomal operon antitermination function. J. Bacteriol. 190, 7251-7257 (2008).

3. Bubunenko, M. et al. Nus transcription elongation factors and RNase III modulate small ribosome subunit biogenesis in Escherichia coli. Mol. Microbiol. 87, 382-393 (2012).

4. Luo, X. et al. Structural and functional analysis of the E. coli NusB-S10 transcription antitermination complex. Mol. Cell 32, 791-802 (2008).

5. Nodwell, J. R. \& Greenblatt, J. Recognition of boxA antiterminator RNA by the E. coli antitermination factors NusB and ribosomal protein S10. Cell 72, 261-268 (1993).

6. Burmann, B. M. et al. A NusE:NusG complex links transcription and translation. Science 328, 501-504 (2010).

7. Prasch, S. et al. RNA-binding specificity of E. coli NusA. Nucleic Acids Res. 37, 4736-4742 (2009)

8. Qayyum, M. Z., Dey, D. \& Sen, R. Transcription elongation factor NusA is a general antagonist of rho-dependent termination in Escherichia coli. J. Biol. Chem. 291, 8090-8108 (2016).

9. Sen, R., Chalissery, J. \& Muteeb, G. Nus factors of Escherichia coli. EcoSalPlus 3, 4.5.3.1 (2008).

10. Li, S. C., Squires, C. L. \& Squires, C. Antitermination of E. coli rRNA transcription is caused by a control region segment containing lambda nut-like sequences. Cell 38, 851-860 (1984).

11. Torres, M., Balada, J. M., Zellars, M., Squires, C. \& Squires, C. L. In vivo effect of NusB and NusG on rRNA transcription antitermination. J. Bacteriol. 186, 1304-1310 (2004)

12. Singh, N. et al. SuhB associates with Nus factors to facilitate $30 \mathrm{~S}$ ribosome biogenesis in Escherichia coli. mBio 7, e00114-e00116 (2016).

13. Peters, J. M. et al. Rho directs widespread termination of intragenic and stable RNA transcription. Proc. Natl. Acad. Sci. USA 106, 15406-15411 (2009).

14. Peters, J. M. et al. Rho and NusG suppress pervasive antisense transcription in Escherichia coli. Genes Dev. 26, 2621-2633 (2012).

15. Inada, T. \& Nakamura, Y. Autogenous control of the suhB gene expression of Escherichia coli. Biochimie 78, 209-212 (1996).

16. Martinez, A., Opperman, T. \& Richardson, J. P. Mutational analysis and secondary structure model of the RNP1-like sequence motif of transcription termination factor Rho. J. Mol. Biol. 257, 895-908 (1996).

17. Bossi, L., Schwartz, A., Guillemardet, B., Boudvillain, M. \& Figueroa-Bossi, N. A role for Rho-dependent polarity in gene regulation by a noncoding small RNA. Genes Dev. 26, 1864-1873 (2012).

18. Wade, J. T. \& Grainger, D. C. Pervasive transcription: illuminating the dark matter of bacterial transcriptomes. Nat. Rev. Microbiol. 12, 647-653 (2014).

19. Mitra, P., Ghosh, G., Hafeezunisa, M. \& Sen, R. Rho proteins: role and mechanisms. Annu. Rev. Microbiol. https://doi.org/10.1146/annurev-micro030117-020432 (2017).

20. Barik, S., Ghosh, B., Whalen, W., Lazinski, D. \& Das, A. An antitermination protein engages the elongating transcription apparatus at a promoter-proximal recognition site. Cell 50, 885-899 (1987).

21. Pasman, Z. \& von Hippel, P. H. Regulation of rho-dependent transcription termination by NusG is specific to the Escherichia coli elongation complex. Biochem. (Mosc.). 39, 5573-5585 (2000).

22. Wilson, H. R., Zhou, J. G., Yu, D. \& Court, D. L. Translation repression by an RNA polymerase elongation complex. Mol. Microbiol. 53, 821-828 (2004).

23. Golomb, M. \& Chamberlin, M. Characterization of T7-specific ribonucleic acid polymerase. IV. Resolution of the major in vitro transcripts by gel electrophoresis. J. Biol. Chem. 249, 2858-2863 (1974).

24. Skinner, G. M., Baumann, C. G., Quinn, D. M., Molloy, J. E. \& Hoggett, J. G. Promoter binding, initiation, and elongation by bacteriophage T7 RNA polymerase. A single-molecule view of the transcription cycle. J. Biol. Chem. 279, 3239-3244 (2004).

25. Burr, T., Mitchell, J., Kolb, A., Minchin, S. \& Busby, S. DNA sequence elements located immediately upstream of the -10 hexamer in Escherichia coli promoters: a systematic study. Nucleic Acids Res. 28, 1864-1870 (2000).

26. Kriner, M. A., Sevostyanova, A. \& Groisman, E. A. Learning from the leaders: gene regulation by the transcription termination factor rho. Trends Biochem. Sci. 41, 690-699 (2016).

27. Sedlyarova, N. et al. sRNA-mediated control of transcription termination in $E$. coli. Cell 167, 111-121 (2016).
28. Wilson, H. R., Kameyama, L., Zhou, J. G., Guarneros, G. \& Court, D. L. Translational repression by a transcriptional elongation factor. Genes Dev. 11, 2204-2213 (1997).

29. Wilson, H. R., Yu, D., Peters, H. K. III, Zhou, J. G. \& Court, D. L. The global regulator RNase III modulates translation repression by the transcription elongation factor N. EMBO J. 21, 4154-4161 (2002).

30. Aksoy, S., Squires, C. L., Squires, C. \& J., B. 1984. Evidence for antitermination in Escherichia coli rRNA transcription. J. Bacteriol. 159, 260-264 (1984).

31. Squires, C. L., Greenblatt, J., Li, J., Condon, C. \& Squires, C. L. Ribosomal RNA antitermination in vitro: requirement for Nus factors and one or more unidentified cellular components. Proc. Natl. Acad. Sci. USA 90, 970-974 (1993).

32. Richardson, E. J. \& Watson, M. The automatic annotation of bacterial genomes Brief. Bioinform. 14, 1-12 (2013).

33. Blattner, F. R. et al. The complete genome sequence of Escherichia coli K-12. Science 277, 1453-1462 (1997).

34. Stringer, A. M. et al. Genome-scale analyses of Escherichia coli and Salmonella enterica AraC reveal non-canonical targets and an expanded core regulon. J. Bacteriol. 196, 660-671 (2014).

35. Stringer, A. M. et al. FRUIT, a scar-free system for targeted chromosomal mutagenesis, epitope tagging, and promoter replacement in Escherichia coli and Salmonella enterica. PLoS ONE 7, e44841 (2012).

36. Chen, Q. \& Kadner, R. J. Effect of altered spacing between uhpT promoter elements on transcription activation. J. Bacteriol. 182, 4430-4436 (2000).

37. Guzman, L. M., Belin, D., Carson, M. J. \& Beckwith, J. Tight regulation, modulation, and high-level expression by vectors containing the arabinose PBAD promoter. J. Bacteriol. 177, 4121-4130 (1995).

38. Edgar, R. C. MUSCLE: multiple sequence alignment with high accuracy and high throughput. Nucleic Acids Res. 32, 1792-1797 (2004).

39. Galperin, M. Y., Makarova, K. S., Wolf, Y. I. \& Koonin, E. V. Expanded microbial genome coverage and improved protein family annotation in the COG database. Nucleic Acids Res. 43, D261-D269 (2015).

\section{Acknowledgements}

We thank Dave Grainger, Don Court, Todd Gray and Keith Derbyshire for helpful discussions and comments on the manuscript. We thank the Wadsworth Center Bioinformatics and Statistics Core Facility, and the Wadsworth Center Media and Tissue Culture Core Facility for technical assistance. This work was supported by the NIH Director's New Innovator Award Program, 1DP2OD007188 (J.T.W.).

\section{Author contributions}

J.T.W., G.B. and N.S. designed the study. J.T.W. and G.B. wrote the manuscript. G.B., N S., C.B., R.J., R.F., M.P., A.M.S. and A.S. generated experimental data. P.L. performed bioinformatic analysis. All authors contributed to data analysis and interpretation.

\section{Additional information}

Supplementary Information accompanies this paper at https://doi.org/10.1038/s41467 017-02124-9.

Competing interests: The authors declare no competing financial interests.

Reprints and permission information is available online at http://npg.nature.com/ reprintsandpermissions/

Open Access This article is licensed under a Creative Commons Attribution 4.0 International License, which permits use, sharing, adaptation, distribution and reproduction in any medium or format, as long as you give appropriate credit to the original author(s) and the source, provide a link to the Creative Commons license, and indicate if changes were made. The images or other third party material in this article are included in the article's Creative Commons license, unless indicated otherwise in a credit line to the material. If material is not included in the article's Creative Commons license and your intended use is not permitted by statutory regulation or exceeds the permitted use, you will need to obtain permission directly from the copyright holder. To view a copy of this license, visit http://creativecommons.org/licenses/by/4.0/.

(C) The Author(s) 2017 